\title{
Momentum-Space Reservoir for Enhancement of Intersubband Second-Harmonic Generation
}

\author{
J. R. Meyer, C. A. Hoffman, F. J. Bartoli, E. R. Youngdale, and L. R. Ram-Mohan
}

\begin{abstract}
We analyze fundamental limits to the secondharmonic conversion efficiency attainable from semiconductor intersubband devices employing asymmetric stepped and double quantum wells. The coupled propagation equations have been solved numerically, accounting for saturation, absorption, and optical heating. It is found that the key figure of merit is the conversion efficiency at the onset of saturation, which has a remarkably simple form depending only on the ratio of broadening time to intersubband relaxation time and on another ratio involving the optical matrix elements. We show that since there are fundamental limits to the values of these ratios, it is unlikely that conversion efficiencies exceeding $\approx 10 \%$ can be attained in devices of the type considered in the previous literature, and for surface incidence even efficiencies approaching that value will require impractically-thick active regions. While detuning from the double resonance condition is often advantageous, net improvements to the optimum performance are relatively modest. However, these limitations can be transcended by placing the subband system in contact with an optically-inactive momentum-space reservoir, which shunts the intersubband relaxation and delays saturation by refilling the depleted subband states with electrons from the reservoir. We propose a specific device based on $\Gamma$-valley active states and L-valley reservoir states in InAs-GaSb-AlSb asymmetric double quantum wells, whose energy levels and optical matrix elements are modeled using an 8-band finite-element calculation. It is predicted that a conversion efficiency of $20 \%$ can be achieved in an active-layer thickness of less than $10 \mu \mathrm{m}$.
\end{abstract}

\section{INTRODUCTION}

$\mathbf{S}$ ECOND-HARMONIC generation phenomena related to intersubband interactions in semiconductor quantum structures have attracted a greatly deal of attention in recent years [1]-[11]. This work has been motivated in part by the need for coherent, high-intensity optical pulses at wavelengths which are not readily available from conventional sources, e.g., large portions of the mid-wave infrared spectral region. Intersubband $\chi_{2 \omega}^{(2)}$ processes are particularly attractive because they have narrow bandwidths and large oscillator strengths, and because the mature growth technologies for material systems such as $\mathrm{GaAs}-\mathrm{Al}_{x} \mathrm{Ga}_{1-x} \mathrm{As}$ and InAs-GaSb-AlSb allow one to exercise considerable flexibility in the band structure engineering of quantum devices. For example, the

Manuscript received April 20, 1994; revised October 20, 1994. This work was supported by The Office of Naval Research.

J. R. Meyer, C. A. Hoffman, and F. J. Bartoli are with the Naval Research Laboratory, Code 5613, Washington, DC 20375 USA.

E. R. Youngdale was with the Naval Research Laboratory, Code 5613 , Washington, DC 20375 USA. He is now with AIB Software, 46030 Manekin Plaza, Suite 160, Dulles, VA 20166 USA.

L. R. Ram-Mohan is with Worcester Polytechnic Institute, Worcester, MA 01609 USA.

IEEE Log Number 9409193 use of stepped wells and asymmetric double wells [4] to break the usual wavefunction symmetries has led to the observation of second-order nonlinearities which are more than 1000 times larger than those attainable from bulk GaAs [6]. It has been predicted [2], [10] that the strength of $\chi_{2 \omega}^{(2)}$ in such structures may allow one to design devices whose secondharmonic conversion length is shorter than the coherence length associated with nonresonant dispersion.

However, in the present work we demonstrate that there are fundamental limits to the maximum second-harmonic conversion efficiencies that one might ultimately hope to attain from optimized quantum-well devices. It will be seen that previous predictions [2], [4], [10] of $\eta_{\max }$ exceeding $30 \%$ are unrealistic when saturation and beam-depletion are properly accounted for. Whereas earlier conclusions were vague because of the large number of material and structural variables involved, we show that, in fact, the most relevant figure of merit is independent of all design parameters except the intersubband relaxation time, the transition linewidth, and the optical matrix elements, which appear in ratio form.

In Sections II and III, we solve the coupled propagation and intersubband-transition equations. Our theoretical approach is similar in spirit to the calculation of DeTemple et al. [12], which was adopted by several subsequent investigators [2], [6], [7], although for clarity we will employ expressions that are more phenomenological in form, and more specialized to the case of intersubband processes in asymmetric quantum wells. It will be demonstrated that the conversion efficiencies for intersubband devices of the types discussed previously are limited to $\approx 10 \%$ under double-resonance conditions, and a very thick active layer $(\gg 10 \mu \mathrm{m})$ is required to attain even that value (we will primarily consider surface incidence, although the conclusions obtained below are easily generalized to the case of waveguide propagation). Section IV treats the effects of detuning from double resonance, which is found to be beneficial but does not dramatically improve the maximum efficiency. It will become apparent that any attempt to circumvent these limitations will probably require a radical modification of the saturation properties. In Section $\mathrm{V}$, we propose to accomplish such a modification by placing the subband system in contact with a "momentum-space reservoir," which shunts the intersubband relaxation and delays saturation by rapidly refilling the depleted subband states with electrons from the optically-inactive reservoir. The specific structure considered is an asymmetric InAs-GaSb-AISb double quantum well, whose doubly-resonant $\Gamma$-valley subbands overlap in energy with a much higher density of L-valley reservoir states. 
The energy levels and optical matrix elements are modeled using an 8-band finite-element calculation. We show that such a structure should yield a much larger conversion efficiency and require a much thinner active region $(<10 \mu \mathrm{m})$ than previous devices employing isolated subband systems which do not interact with a reservoir.

\section{CONVERSion EFFiciency at SATURATION AND SOLUTION OF THE PROPAGATION EQUATIONS}

It is well known that in the small-signal limit for which one may ignore the effects of beam depletion and saturation, the second harmonic conversion efficiency is given (in cgs units) by [13]

$$
\eta=\frac{128 \pi^{3}}{n \kappa c^{3}}\left[\omega \chi_{2 \omega}^{(2)} L^{\prime}\right]^{2} \frac{\sin ^{2}\left(\pi L^{\prime} / L_{c}\right)}{\left(\pi L^{\prime} / L_{c}\right)^{2}} I_{1}
$$

where $n$ is the index of refraction, $\kappa$ is the dielectric constant, $c$ is the speed of light, $\omega$ is the photon frequency of the pump beam, $I_{1}$ is the intensity of the pump beam, $L_{c}$ is the coherence length, $L^{\prime}=L / \cos \theta$ is the propagation length, $L$ is the thickness of the active layer, and $\theta$ is the internal angle of incidence. In the analysis that follows, we will take the propagation length to be much shorter than the coherence length, although the restrictions imposed by this assumption will later be reexamined. For a three-level system, the secondharmonic generation coefficient is given by [13]

$$
\chi_{2 \omega}^{(2)} \approx \frac{F n^{2} e^{3} S_{2} N z_{12} z_{13} z_{23} \sin ^{3} \theta}{\kappa\left(\hbar \omega-E_{12}-i \hbar \Gamma_{12}\right)\left(2 \hbar \omega-E_{13}-i \hbar \Gamma_{13}\right)} .
$$

Here $F$ is the fill factor (ratio of quantum-well thickness to total thickness of each period), $S_{2}\left(I_{1}, I_{2}\right)$ is a saturation factor which approaches unity at low excitation levels (see below), $I_{2}$ is the intensity of the second-harmonic beam, $N$ is the threedimensional electron concentration in each quantum well, $E_{i j}$ is the energy separation between subbands $i$ and $j$, and $\hbar \Gamma_{i j}$ is the linewidth associated with a given transition (we will hereafter make the usual assumption that all transitions have equal $\Gamma$ ). The dipole matrix element for optical transitions between subbands $i$ and $j$ is $z_{i j} \sin \theta$, where $z_{i j} \equiv\langle i|z| j\rangle$ is its value for waveguide geometry. It is well known that if the conduction-band minimum for the quantum well system is at the spherically-symmetric $\Gamma$-point, as has been the case in all of the experimental intersubband $\chi^{(2)}$ studies reported to date $[14],{ }^{1}$ the matrix elements vanish for normal incidence.

Saturation of the second-harmonic generation occurs whenever the intensity is high enough that intersubband absorption, which takes place in parallel with frequency conversion, depopulates the quantum well ground state [7]. We define the saturation intensity as

$$
I_{1 s}=\frac{F N \hbar \omega}{2 \alpha_{12}^{0} \tau_{12}} .
$$

where $\tau_{12}$ is the intersubband relaxation time, $\alpha_{i j}$ is the absorption coefficient for $i \rightarrow j$ transitions, and $\alpha_{i j}^{0}$ is its

${ }^{1}$ For a theoretical treatment of normal incidence ${ }^{(2)}$ processes associated with $L$-valley quantum wells, see [14] value in the low-intensity limit. We may write [15]

$\alpha_{i j}\left(\omega^{\prime}\right)=\frac{4 \pi n e^{2} F S_{\alpha}^{i j} N \hbar \omega_{i j} z_{i j}^{2} \sin ^{2} \theta}{\kappa \hbar c} \frac{\hbar \Gamma}{\left(\hbar \omega^{\prime}-E_{i j}\right)^{2}+(\hbar \Gamma)^{2}}$,

where $S_{\alpha}^{i j}\left(I_{1}, I_{2}\right) \equiv \alpha_{i j} / \alpha_{i j}^{0}=\left(N_{i}^{i}-N_{j}\right) / N$ is the saturation factor for absorption and $N_{i}$ is the intensity-dependent density of electrons populating a given subband ( $N=N_{1}+N_{2}+$ $N_{3}$ if only the first three levels are considered). Because absorption depletes the pump beam, the optimum propagation length for maximizing the conversion efficiency tends to be on the order of the absorption depth. Hence,

$$
L_{\max }\left(I_{1} \leq I_{1 s}\right) \approx L_{\max }^{0} \equiv \cos \theta / \alpha_{12}^{0}(\omega) .
$$

From (2.2), it is apparent that the second-harmonic generation is strongest when there is a double resonance between the photon energy and the intersubband energy separations, i.e., $\hbar \omega=E_{12}=E_{23}$ and hence $2 \hbar \omega=E_{13}$. Combining (2.1)-(2.5) under double-resonance conditions (the consequences of detuning from resonance will be discussed in Section IV), we can estimate the maximum conversion efficiency attainable at the onset of saturation. For $I_{1} \rightarrow I_{1 s}$ and taking $S_{2} \approx S_{\alpha}^{i j} \approx 1$ (since the saturation is not yet severe), we obtain

$$
\eta_{s}=\frac{\tau_{\Gamma}}{\tau_{12}} R_{12},
$$

where $\tau_{\Gamma} \equiv 1 / \Gamma$ is the broadening time and

$$
R_{12} \equiv\left(\frac{z_{23} z_{13}}{z_{12}^{2}}\right)^{2}
$$

This remarkably simple result, which to our knowledge has not been discussed previously (although equation (50) in [4] is somewhat related), is seen to contain no explicit dependences on most of the usual design parameters, including angle of incidence, fill factor, doping density, refractive index, or photon frequency. Furthermore, even the broadening time, relaxation time, and matrix elements enter only in ratio form. While final optimization of the second-harmonic generation will be seen to require excitation at pump intensities considerably exceeding $I_{1 s}$, it will nonetheless become apparent that $\eta_{s}$ remains the most important figure of merit governing the ultimate limits to the conversion efficiency.

In order to consider intensities beyond the saturation threshold, it becomes necessary to numerically solve the coupled differential equations for propagation of the pump and secondharmonic beams. As in the calculation of DeTemple et al. [12], but not in the recent treatment of Rosencher [10], we will account for saturation of the absorption as well as saturation of the second-harmonic generation, where the double-resonance form [8], [12] $S_{2} \approx\left(N_{1}-2 N_{2}+N_{3}\right) / N$ will be used. Employing the normalized variables $a_{k} \equiv\left(I_{k} / I_{1 s}\right)^{1 / 2}(k=1$ for the pump beam and $k=2$ for the second-harmonic beam), and $s \equiv \alpha_{12}^{0}(\omega) z / \cos \theta$ ( $z$ is the depth from the sample surface), we have for the propagation equations [16]

$$
\frac{d a_{1}}{d s}=-\left[\frac{\alpha_{12}(\omega)+\alpha_{23}(\omega)}{\alpha_{12}^{0}(\omega)}\right] \frac{a_{1}}{2}-S_{2} \eta_{s}^{1 / 2} a_{1} a_{2}
$$


and

$$
\frac{d a_{2}}{d s}=-\left[\frac{\alpha_{13}(2 \omega)}{\alpha_{12}^{0}(\omega)}\right] \frac{a_{2}}{2}+S_{2} \eta_{s}^{1 / 2} a_{1}^{2},
$$

whose boundary conditions are fixed by the input intensities of the pump beam $\left[I_{1}(s=0)=I_{1}^{\text {in }}\right]$ and second-harmonic beam $\left[I_{2}(s=0)=0\right]$. It was pointed out above that the saturation factors $S_{\alpha}^{i j}$ and $S_{2}$ depend on the relative populations of the three subbands at given $I_{1}(s)$ and $I_{2}(s)$. These may be obtained from the steady-state solutions to the coupled rate equations at each depth $s$ :

$$
\begin{aligned}
\frac{d N_{1}}{d t}=-\frac{\alpha_{12} I_{1}(s)}{\hbar \omega}- & \frac{\alpha_{13} I_{2}(s)}{2 \hbar \omega} \\
& +\frac{N_{2}-N_{20}}{\tau_{12}}+\frac{N_{3}-N_{30}}{\tau_{13}} \rightarrow 0
\end{aligned}
$$

and

$$
\begin{aligned}
\frac{d N_{2}}{d t}=\frac{\alpha_{12} I_{1}(s)}{\hbar \omega}- & \frac{\alpha_{23} I_{1}(s)}{\hbar \omega} \\
& -\frac{N_{2}-N_{20}}{\tau_{12}}+\frac{N_{3}-N_{30}}{\tau_{23}} \rightarrow 0,
\end{aligned}
$$

where $N_{3}=N-N_{1}-N_{2}, N_{30}=N-N_{10}-N_{20}$, and $N_{i 0}$ is the concentration of a given level in thermal equilibrium:

$$
N_{10}=\frac{N}{1+e^{-E_{12} / k_{B} T_{\mathrm{e}}}+e^{-E_{13} / k_{B} T_{\mathrm{e}}}}
$$

and

$$
N_{20}=\frac{N e^{-E_{12} / k_{B} T_{e}}}{1+e^{-E_{12} / k_{B} T_{e}}+e^{-E_{13} / k_{B} T_{e}}}
$$

for nondegenerate statistics. While $N_{20}$ and $N_{30}$ vanish in the limit of low electron temperatures (i.e., when $k_{B} T_{e} \ll E_{12}$ ), optical heating of the electron bath should be considered at high excitation levels. Equating the energy gain and loss rates gives

$$
k_{B} T_{e}=k_{B} T+\frac{\hbar \omega}{3}\left(\frac{\tau_{E}}{\tau_{12}}\right) \frac{\left(\alpha_{12}+\alpha_{23}\right) I_{1}+\alpha_{13} I_{2}}{\alpha_{12}^{0} I_{1 s}},
$$

where $T$ is the lattice temperature, $\tau_{E} \approx\left(2 \hbar \omega_{\mathrm{PO}} / 3 \hbar \omega\right) O_{12} \tau_{12}$ is the intrasubband energy relaxation rate, [17] $\hbar \omega_{\mathrm{PO}}$ is the optical phonon energy, and $O_{12}$ is a normalized integral quantifying the spatial overlap of the wavefunctions for the first two subbands. We emphasize that the magnitude of $\mathrm{O}_{12}$ is closely correlated with that of the optical matrix element $z_{12}$. For $\hbar \omega$ corresponding to $\mathrm{CO}_{2}$ wavelengths and relatively strong coupling between the first two subbands $\left(\mathrm{O}_{12} \approx 1\right)$, this relation yields: $\tau_{E} / \tau_{12} \approx 0.2$ (roughly consistent with experimental results for $\tau_{E}$ and $\tau_{12}$ in the case of square wells [18]-[20]), although the ratio will be somewhat smaller if the coupling is weak $\left(O_{12}<1\right)$. With this relaxation time ratio, (2.14) yields $k_{B} T_{e} / E_{12} \ll 1$ at the saturation intensity, for which the equilibrium population of the excited subbands is negligible. The numerical calculations further show that even at intensities well above $I_{1 s}$, the electron thermal energy tends to level off at a value on the order of $0.1 E_{12}$. Thus the thermal redistribution of the electron population plays a relatively modest role in limiting $\eta_{\max }$. The additional influence of

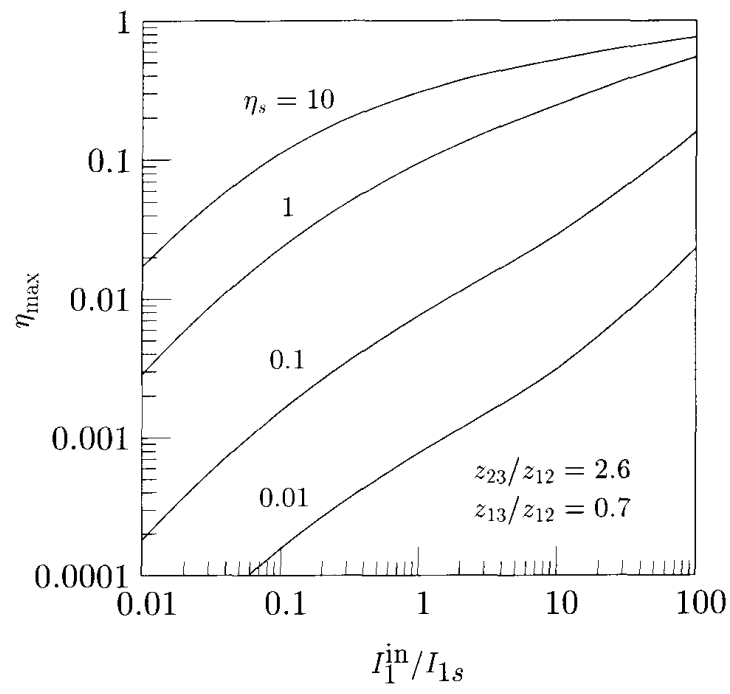

Fig. 1. Maximum conversion efficiency vs incident intensity at several values of $\eta_{s}$, for devices of optimum thickness. The matrix-element ratios employed in the calculations are indicated.

$\Delta T_{e} \approx 100 \mathrm{~K}$ on the broadening time that appears in (2.6) will be discussed below.

Using (2.10)-(2.14), to evaluate the depth-dependent subband populations corresponding to the intensities $I_{1}(s)$ and $I_{2}(s)$, we have solved the propagation equations (2.8) and (2.9) numerically for a wide range of input parameters. For $\eta_{s}$ values spanning three orders of magnitude, Fig. 1 plots typical results for the maximum conversion efficiency (i.e., evaluated at the optimum thickness $\left.L_{\max }\left(I_{1}^{\text {in }}\right)\right)$ as a function of input intensity. These results were obtained assuming $\tau_{12} / \tau_{23} \rightarrow 1$ and $\tau_{12} / \tau_{13} \rightarrow 0$, and employing the matrixelement ratios $z_{23} / z_{12}=1.62$ and $z_{13} / z_{12}=0.85$, which are appropriate for the asymmetric double well considered by Rosencher [10] (that structure may be viewed as nearly optimal). If one ignores absorption of the second-harmonic beam, the propagation equations yield the asymptotic form $\eta_{\max } \rightarrow \eta_{s} I_{1}^{\text {in }} / I_{1 s}$ in the limit $I_{1}^{\text {in }} \rightarrow 0$. However, comparison of this expression with low-intensity values in the figure indicates that $\alpha_{13}$ transitions have reduced the proportionality constant somewhat. As expected, saturation of the nonlinearity due to population of the higher subbands causes the slope to decrease once the input intensity approaches a value on the order of $I_{1 s}$ (see in particular the curves for $\eta_{s}=0.01$ and 0.1 ). However, a second saturation mechanism becomes important at larger $\eta_{s}$, namely depletion of the input beam by frequency conversion [16]. This is apparent in the curves for $\eta_{s}=1$ and 10 , whose slopes are seen to decrease at $I_{1}^{\text {in }}$ somewhat below $I_{1 s}$. Although details of the function $\eta_{\max }\left(I_{1}^{\mathrm{in}}\right)$ naturally depend on the particular values chosen for the matrix-element and intersubband-relaxation-time ratios, the crucial importance of maximizing $\eta_{s}$ should be apparent from this figure.

It was pointed out above that while $L_{\max }^{0}$ on the order of $\cos \theta / \alpha_{12}^{0}$ tends to be optimum at low intensities, saturation of the absorption allows one to employ a somewhat thicker active region at intensities above $I_{1 s}$ [2], [4], [5], [12]. This is 


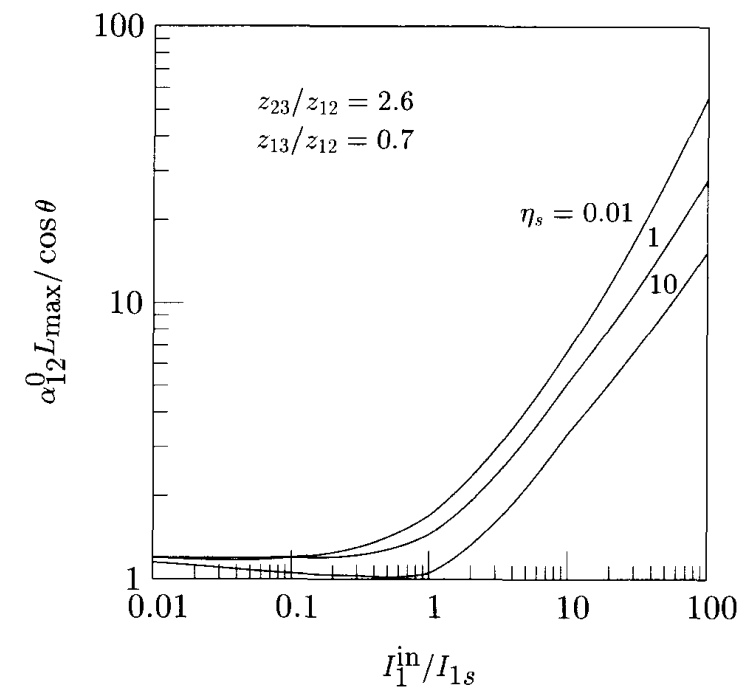

Fig. 2. Optimum thicknesses (normalized by the low-intensity absorption depth) vs. incident intensity, for several $\eta_{s}$.

illustrated in Fig. 2, which plots the optimum sample thickness as a function of $I_{1}^{\text {in }}$. While $L_{\max }$ is nearly constant at low intensities, it is seen to increase rapidly once the saturation threshold is reached, following a functional form which is relatively independent of $\eta_{s}$. Relation to the coherence length and practical limitations connected with the growth will be discussed below.

\section{LIMITS TO CONVERSION EFFICIENCY - DOUBLE RESONANCE}

It should be evident from the discussion in the preceding section that three primary factors govem the upper bound on the second-harmonic conversion efficiency attainable from optimized intersubband-based devices.

1) Maximum Input Intensity: While other practical considerations may play a role, the maximum intensity is ultimately limited by plasma breakdown at the surface of the sample. Since the breakdown intensity tends to be relatively independent of material for comparable surface preparations, we will use the value $\approx 60 \mathrm{MW} / \mathrm{cm}^{2}$ which is appropriate for GaAs-based devices [21].

2) Maximum Active-Region Thickness: The maximum $L$ is ultimately limited by the loss of coherence (which is ignored in the calculations discussed above). Since $L_{c} \approx 100 \mu \mathrm{m}$ for GaAs-based structures at pump-beam wavelengths on the order of $10 \mu \mathrm{m}$ [22], the coherence factor in (2.1) implies that $\eta$ degrades by more than a factor of 2 for any propagation length $(L / \cos \theta)$ exceeding $\approx 50 \mu \mathrm{m}$. An additional limitation is the slow deposition rate associated with molecular beam epitaxy (MBE), which makes it impractical to employ thicknesses exceeding $\approx 10 \mu \mathrm{m}$ (accurately controlling the substrate temperature, and hence the quantum-well layer thicknesses, also becomes an issue in the case of very long growth runs). While the growth rate is somewhat faster for metalorganic chemical vapor deposition (MOCVD), accurate control over the asymmetric-well layer thicknesses becomes much more difficult using that process. To our knowledge, all experimental intersubband $\chi_{2 \omega}^{(2)}$ studies reported to date have employed MBE materials. The rapid increase of the required sample thickness following saturation (see Fig. 2) make it particularly important to optimize the conversion efficiency be fore saturation occurs, i.e., to maximize $\eta_{s}$.

3) Magnitude of $\eta_{s}$ : In evaluating $\eta_{s}$ from (2.6), we first consider the broadening time, $\tau_{\Gamma}$. While discussions of intersubband-based nonlinear processes often refer to this parameter as the dephasing time, in practice it includes broadening due to nonparabolicity and sample nonuniformity in addition to the scattering contribution. The absorption linewidth in the best materials at low temperatures tends to be dominated by nonparabolicity, which leads to $\hbar \Gamma \geq 3$ meV in GaAs and $\mathrm{In}_{1-x} \mathrm{Ga}_{x}$ As quantum wells with doping levels in the range of interest [23], [24] (narrower widths could in principle be attained in lightly-doped materials [25]). With increasing temperature, the broadening contribution due to optical phonon scattering becomes significant. This leads to a minimum linewidth of $\approx 4 \mathrm{meV}$ at $300 \mathrm{~K}$, [23], [24], [26], [27], which corresponds to $\tau_{\Gamma}=0.16 \mathrm{ps}$ (previous calculations based on longer dephasing times are therefore physically unreasonable). We emphasize that even this value should be considered somewhat optimistic, since $\hbar \Gamma \geq 7$ $\mathrm{meV}\left(\tau_{\Gamma} \leq 0.9 \mathrm{ps}\right)$ is more typical of "good" quantum-well material at $300 \mathrm{~K}$. Note also that since $\tau_{\Gamma}$ depends relatively weakly on temperature, there is little incentive to operate the second-harmonic-generation device at low $T$.

Since a short intersubband relaxation time leads to a high saturation intensity [7], we wish to minimize $\tau_{12}$ (note that for intersubband lasers [28], one has the opposite goal of maximizing $\tau_{12}$ ). Scattering theory yields that the intersubband relaxation rate scales roughly as [17], [29]

$$
\tau_{12}^{-1} \propto m_{z} d_{W} O_{12} \propto\left(m_{z} / \hbar \omega\right)^{1 / 2} O_{12}
$$

where $m_{z}$ is the bulk effective mass along the confinement axis, $d_{W}$ is the quantum well thickness, and the latter expression uses the resonance relation: $\hbar \omega \approx E_{i j} \propto$ $1 /\left(m_{z} d_{W}^{2}\right)$. Equation (3.1) implies that while it is slightly advantageous to employ a material with larger effective mass, the strongest variation is related to geometry, through $O_{12}$. Experimental intersubband relaxation times reported for GaAs and $\mathrm{In}_{1-x} \mathrm{Ga}_{x}$ As square wells cover a broad range of values, ranging between 0.5 ps and 15 ps [7], [30], [21], [31], [19], [32], [20]. Many of the longer values have been attributed to either inadequate confinement of the upper level or trapping within the barrier [33], [34] (both may be considered manifestations of weak overlap). Sun and Khurgin [35] derived the relation $\left.O_{12} \propto q_{z}^{2} z_{12}^{2}\left(1+q_{z}^{2} z_{12}^{2} / 2\right)\right)$, where to obtain the net scattering rate one integrates over the growth-direction wavevector $q_{z}$. Thus $\tau_{12}$ becomes quite long (e.g., $18 \mathrm{ps}$ ) in asymmetric double wells with wide separation distances and hence weak $O_{12}$ (small $z_{12}$ ) [8]. Combining the results for $\tau_{\Gamma}$ and $\tau_{12}$, we find that in quantum well structures of the type considered thus far, one is unlikely to achieve $\tau_{\Gamma} / \tau_{12}$ exceeding $\approx 0.15$. 
Turning now to the matrix-element ratio which appears in (2.6), we note that for a stepped quantum well, $\chi_{2 \omega}^{(2)} \propto$ $z_{12} z_{13} z_{23}$ is maximized when the steps are of approximately equal width (see Rosencher and Bois [8, Fig. 4]). However, such structures tend to have small $z_{13} / z_{12}$, and hence small $R_{12}$ (e.g., $\approx 0.07$ for the case considered in [8, Fig. 11], which yields $\eta_{s}<0.01$ ). By increasing the width of the shallower step one can increase $R_{12}$ [14], although values on the order of unity probably represent a practical limit for stepped wells. On the other hand, in an asymmetric double well one can make $z_{12}$ far weaker than $z_{13}$ (and hence $R_{12}$ arbitrarily large) by increasing the spatial separation of the two wells. However, there are several factors which limit this strategy. First, Rosencher has pointed out that it is disadvantageous for the ratio $z_{13} / z_{12}$ to be larger than the order of unity [10], since absorption of the second-harmonic beam then becomes a dominant mechanism. It is easily shown that when $z_{13} / z_{12} \gg$ 1 , (2.5) should be replaced by $L_{\max } \rightarrow \cos \theta / \alpha_{13}^{0}$ and the saturation comes to be governed by $\alpha_{13}$ absorption, implying that the ratio $R_{12}$ in (2.6) should be replaced by $R_{13} \equiv$ $\left(z_{12} z_{23} / 2 z_{13}^{2}\right)^{2}$. Furthermore, a significant weakening of $z_{12}$ will unavoidably be accompanied by a longer intersubband relaxation time [8] (due to the smallness of $O_{12}$ ), as well as by the requirement for a much thicker active layer. It is therefore difficult to achieve $R_{12}$ greater than $\approx 3$ in any optimized stepped-quantum-well or asymmetric-double-well structure. Combining this estimate with that for the $\tau_{\Gamma} / \tau_{12}$ ratio obtained above, we conclude that the limit $\eta_{s} \leq 0.45$ represents a nearly absolute maximum for intersubband nonlinearities of the type discussed in the previous literature. Figs. 1 and 2 indicate that the achievement of quantum efficiencies significantly exceeding $10 \%$ would then require $I_{1}^{\text {in }} / I_{1 s}$ approaching 100 (which would generally induce plasma breakdown) and $L$ more than 10 times the low-intensity absorption depth.

We illustrate by treating the $\mathrm{In}_{1-x} \mathrm{Ga}_{x} \mathrm{As}-\mathrm{GaAs}-\mathrm{Al}_{x} \mathrm{Ga}_{1-x}$ As double-well structure discussed theoretically by Rosencher [10], which may be considered nearly optimal. Using $z_{12}=13$ $\AA, z_{13}=11 \AA, z_{23}=21 \AA$, and $\hbar / \tau_{\Gamma}=10 \mathrm{meV}$ from that reference and taking $\tau_{12}^{1}=1 \mathrm{ps}$, we obtain $R_{12}=1.9$ and $\eta_{s}=0.22$. For $\theta=45^{\circ},(2.3)$ and (2.4) imply a saturation intensity of $3.4 \mathrm{MW} / \mathrm{cm}^{2}$ and hence a maximum intensity ratio before plasma breakdown of $I_{1}^{\text {in }} / I_{1 s} \approx 17.5$. For these input parameters, the numerical solution to the propagation equations yields $\eta_{\max } \approx 0.085$, which is nearly a factor of 4 smaller than the value obtained in [10] when saturation was neglected. Furthermore, using the absorption depth of $6.8 \mu \mathrm{m}$ at $45^{\circ}$ incidence [10], we find that the active layer thickness required to achieve the maximum conversion efficiency is 49 $\mu \mathrm{m}$, i.e., great enough to induce significant loss of coherence and also impractical from a fabrication standpoint. While longer propagation lengths are easily attainable in waveguide devices [11], loss of coherence remains a significant limitation.

We conclude that previous predictions [2], [10] of $>30 \%$ conversion efficiency from conventional 3-level doubleresonance quantum well devices are unrealistic. Our finding is consistent with the analysis of DeTemple et al. [12], who obtained $\eta_{\max }$, exceeding $50 \%$, but only in the limit $\tau_{12} \rightarrow \tau_{\Gamma}$, which does not apply to the intersubband nonlinearities considered here. To our knowledge, the largest $\eta$ reported experimentally thus far is $0.3 \%$ [11].

\section{LIMITS TO CONVERSION EFFICIENCY- DETUNING FROM DOUBLE RESONANCE}

It has been suggested that higher maximum conversion efficiencies can be achieved by detuning the photon energy from resonance with the intersubband energy separations [4], [5], since the benefit of reduced absorption losses may outweigh the smaller second-harmonic generation coefficient which results $\left(\left(\chi^{(2)}\right)_{2 \omega}^{2} /\left(\alpha_{12}^{0}\right)^{3}\right.$ displays a net increase).

We first consider the single-resonance case, in which the condition $2 \hbar \omega-E_{13}=0$ is retained while setting $\hbar \omega-E_{12}=$ $-\left(\hbar \omega-E_{23}\right) \equiv \delta$. As compared to the values for double resonance, $\chi_{2 \omega}^{(2)}$ is now smaller by a factor of

$$
\beta \equiv \frac{\hbar \Gamma}{\left[\delta^{2}+(\hbar \Gamma)^{2}\right]^{1 / 2}} .
$$

$\alpha_{12}$ and $\alpha_{23}$ are smaller by factors of $\beta^{2}, \alpha_{13}$ is unchanged, and $I_{1 s}$ is larger by a factor of $\beta^{-2}$. When these results are combined we obtain $\eta_{s} \rightarrow \eta_{s} / \beta^{2}$, which potentially represents a large net increase. However, this enhancement is difficult to realize in practice, since with $z_{13} \approx z_{12}$ the absorption of the second-harmonic beam soon becomes the dominant mechanism limiting the maximum layer thickness (i.e., it does no good to decrease $\alpha_{12}$ beyond a certain point). Numerically solving the propagation equations under singleresonance conditions confirms that with $\beta=0.5$ in the structure used as an example in the previous section [10], the maximum conversion efficiency increases only slightly, from $8.5 \%$ to $9.7 \%$ (further decreases of $\beta$ eventually lead to a decrease of $\eta$ ). While the detuning from double resonance also allows one to employ a slightly thinner active layer (37 $\mu \mathrm{m}$ rather than $49 \mu \mathrm{m}$ ), these improvements are relatively modest.

While absorption of the second-harmonic beam plays a dominant role under single-resonance conditions, we can minimize this effect by detuning from resonance with the $2 \hbar \omega$ transitions as well. Assuming for definiteness that both detuning energies are equal $\left(\hbar \omega-E_{12}=2 \hbar \omega-E_{13} \equiv \delta\right)$, we now obtain that $\chi_{2 \omega}^{(2)}$ is decreased by a factor of $\beta^{2}, \alpha_{12}$ and $\alpha_{13}$ are smaller by factors of $\beta^{2}, \alpha_{23}$ is unchanged, and $I_{1 s}$ is larger by a factor of $\beta^{-2}$. Combining, we find $\eta_{s} \rightarrow \eta_{s} / \beta$. As long as full coherence is assumed, the propagation equations yield a steady increase of $\eta_{\max }$ with decreasing $\beta$, reaching $\eta_{\max }=16 \%$ at $\beta=0.5$ and $18 \%$ at $\beta=0.3$. However, this is accompanied by a gradual increase of the required sample thickness (to $52 \mu \mathrm{m}$ at $\beta=0.5$ and $73 \mu \mathrm{m}$ at $\beta=$ 0.3 ), making the loss of coherence an even more significant limitation than in the double-resonance case.

Thus, while final optimization of the conversion efficiency will probably involve some degree of detuning from either one or both of the resonances, it is unlikely that conventional 3level quantum-well devices with practical active-region thicknesses will ever yield $\eta_{\max }$ significantly exceeding $\approx 10 \%$. In the next section, we propose to circumvent this limitation 
by placing the optically-active intersubband system in contact with a reservoir.

\section{EFFICIENCY ENHANCEMENT VIA INCORPORATIONS OF A MOMENTUM-SPACE RESERVOIR}

Fundamental limits to the second-harmonic conversion efficiency are to a large extent governed by the saturation properties. The rapid saturation discussed above is in turn directly attributable to the inefficiency of the intersubband relaxation process, which unavoidably yields $\tau_{12} \geq 10 \tau_{\Gamma}$ for isolated 3-level subband systems produced by current technology. While it would be difficult to increase the relaxation rate for the usual phonon mechanism (although it can be made slower by decreasing $O_{12}$ ), there are nonetheless artificial means by which one can attempt to reduce the effective $\tau_{12}$. Here, we consider an approach based on placing the subband system in contact with a high-density reservoir of opticallyinactive states, whose function is to provide elastic scattering into and out of the active subband states. Thus, when an $\alpha_{12}$ or $\alpha_{13}$ absorption process excites an electron out of the $i=1$ subband, that carrier is rapidly replaced by an electron from the reservoir. This refills the state on a much faster time scale than would ordinarily be possible in the isolated subband system. Similarly, electrons excited to the $E_{2}$ and $E_{3}$ levels quickly scatter into reservoir states at those higher energies. Energy relaxation then takes place within the reservoir, which can in principle be much faster than in the isolated subband system. Furthermore, even though the optical transitions involve only subband states, the replenishment by reservoir electrons delays the onset of saturation (which does not occur until the entire lower-energy portion of the reservoir is emptied). It is naturally essential that the reservoir states be optically-inactive, so that intra-reservoir and reservoir-subband absorption processes do not significantly deplete the pump and second-harmonic beams.

In one configuration, spatial reservoirs could be placed on each side of the active asymmetric region. For example, these could consist of thick quantum wells with ground-state energies near $E_{1}$ and a number of closely-spaced subbands below the Fermi energy. The density of reservoir electrons would then be much greater than the density of active-region electrons, considerably increasing the saturation threshold. Higher-energy reservoir levels would also provide a high density of states for elastic scattering from the $E_{2}$ and $E_{3}$ active-region subbands. Energy relaxation within the reservoir will be rapid, due to the close spacing of the reservoir subbands (see (3.1)). While intra-reservoir optical transitions should be weak due to the large $\Delta i$ corresponding to the relevant $\hbar \omega$, one must take care to insure that the optical coupling between reservoir states and active-region subbands is also weak. Unfortunately, the fill factor for such a device will be rather small, due to the large ratio of reservoir-region thickness to active-region thickness.

Probably a more attractive alternative is to introduce a reservoir in momentum space. As an explicit example, we consider an InAs-GaSb-AlSb double-well structure, in which the $\Gamma$-point conduction-band minimum is at only slightly lower energy than the minima for the four degenerate $L$ point valleys. While the design of the asymmetric wells is such that the $\Gamma$-point subbands are doubly resonant and have favorable optical matrix elements, the $L$ valleys are ideally suited to serving as a reservoir. Their 2-D density of states is roughly 30 times that of the $\Gamma$ valley, yet they can be made optically inactive by detuning the intersubband spacings to values far from resonance with the pump and second-harmonic photon energies. Measured $\mathrm{L}-\Gamma$ intervalley relaxation times for GaAs-based alloys and heterostructures tend to be extremely short, falling in the 50-200 fs range [36]-[39]. The intervalley transfer is dominated by LA and LO phonon scattering, and it has been shown theoretically that the $\Gamma$-valley intersubband relaxation rate can be increased by shunting through $L$-valley states [34], [40]. A further advantage is that since the intersubband relaxation now occurs primarily through the $L$-valley, it has become largely decoupled from the overlap integral $O_{12}$. That is, one has far greater flexibility to modify $z_{12}$ without adversely affecting $\tau_{12}$. Although $L$-valley scattering may broaden the $\Gamma$-valley transition linewidth (by as much as a factor of 2 if the intervalley relaxation time is very short), this effect is suppressed because the $\Gamma$-valley conduction band minimum will lie somewhat lower than the $L$-valley minimum. At any rate, the ratio $\tau_{\Gamma} / \tau_{12}$ should show a net increase. We do not expect the intermixing of $\Gamma$ and $L$ states to have an appreciable effect on the dipole matrix elements. For the analogous case of $\Gamma-X$ interactions in the GaAs- $\mathrm{Al}_{x} \mathrm{Ga}_{1-x} \mathrm{As}$ system, it has been found that the oscillator strengths for both interband and intersubband transitions between $X$-states and $\Gamma$-states are 4-7 orders of magnitude smaller than those for $\Gamma-\Gamma$ transitions [41], [42].

An example of a suitable asymmetric structure is illustrated in Fig. 3. For the same spatial region containing the double well, the (a) and (b) portions of the figure give conductionband profiles for the $\Gamma$ and $L$ valleys, respectively. The sequence consists of a relatively-thick AlSb barrier layer (e.g., $100 \AA$ ), a $51-\AA$ GaSb quantum well, a 2-monolayer (6.14 $\AA$ ) AlSb barrier, a $25-\AA$ GaSb layer, a 1-monolayer (3.03- $\AA$ ) InAs layer, a $50-\AA$ GaSb layer, and finally a repeat of the thick AlSb barrier which separates this double well from the next period. Although some of the layers are quite thin, the energy levels and matrix elements are not extremely sensitive to the precise thicknesses, and the all-binary construction is advantageous from a growth standpoint.

Quantized energy levels, wavefunctions, and optical matrix elements for this structure at the $\Gamma$-point have been calculated using an 8-band finite-element $(k \cdot p)$ formalism [43], which allows for an arbitrary number of constituent layers with arbitrary band profiles. Since the $k \cdot p$ method is inapplicable to systems with ellipsoidal constant-energy surfaces, results for the $L$-valley subbands were obtained from a 1-band version of the finite-element algorithm (the second-harmonic generation is not nearly as sensitive to the details of the $L$ valley properties). That calculation employed $L$-point band profiles and quantization masses for each constituent material $\left(m_{z}=3 m_{t} m_{l} /\left(m_{t}+2 m_{l}\right)\right.$ for [100] growth [44], where $m_{t}$ and $m_{i}$ are the $L$-valley transverse and longitudinal masses). Most of the bulk input parameters were taken from [45], 


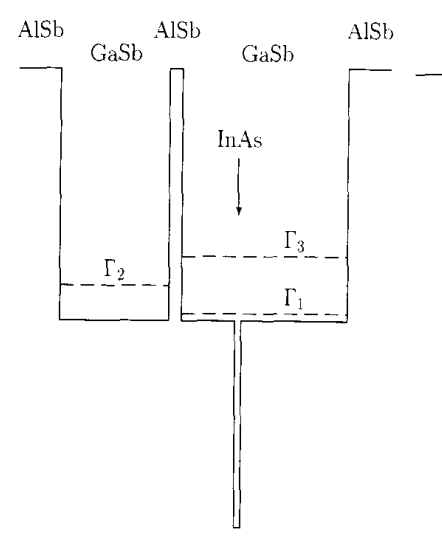

(a) (b)
Fig. 3. $\Gamma$-point (a) and L-point (b) conduction band profiles and energy levels for asymmetric double quantum well with momentum-space reservoir

valence band offsets were from [46] and [47], and the $L$ valley minima were taken to lie $40 \mathrm{meV}$ above the $\Gamma$-valley minimum in bulk $\mathrm{GaSb}$ at $300 \mathrm{~K}$ [48]. The primary purpose of the thin InAs layer is to assure that the energy of the lowest $\Gamma$-point subband is slightly below that of the lowest $L$ point subband. The same thing can be accomplished by adding a small concentration of In to the GaSb well on the right, i.e., by employing an AlSb-GaSb-AlSb-Ga $a_{1-x} \operatorname{In}_{x} \mathrm{Sb}-\mathrm{AlSb}$ double quantum well. Note also that whereas the InAs $\Gamma$-point minimum lies far below the $\Gamma$-point in GaSb, at the $L$-point the InAs contributes a shallow barrier within the $\mathrm{GaSb}$ quantum well.

The dashed curves in Fig. 3 indicate energy minima for the various $\Gamma$-point and $L$-point subbands, where the location of a given line specifies whether the wavefunction for that subband is concentrated in the thinner quantum well at left or in the thicker well at right. The structure is designed such that the $\Gamma$-point subband splittings are in double resonance with a pump-beam photon energy of $122 \mathrm{meV}$. The optical matrix elements calculated for the $\Gamma$ levels are $z_{12}=16.1 \AA, z_{13}=$ $17.0 \AA$, and $z_{23}=25.1 \AA$, which yield $R_{12}=2.7$. Since the two lowest $\mathrm{L}$-point subbands have energies only slightly above the $\Gamma$-point minimum $\left(E_{L 1}-E_{\Gamma 1}=39 \mathrm{meV}\right.$ and $E_{L 2}-$ $\left.E_{\Gamma 1}=58 \mathrm{meV}\right)$ and their densities of states are much higher, roughly $90 \%$ of the electrons reside in the L-point reservoir at low optical intensities. Nonetheless, the modeling yields that the pump-beam and second-harmonic-beam absorption coefficients due to $L$-point transitions are less than $10 \%$ of $\alpha_{12}$ and $\alpha_{13}$, since the intersubband splittings are far out of resonance with both $\hbar \omega$ and $2 \hbar \omega$ (e.g., $E_{L 3}-E_{L 1}=75 \mathrm{meV}$ and $E_{L 4}-E_{L 2}=153 \mathrm{meV}$ ). For both $\Gamma$-point and $L$-point interactions, we have assumed energy linewidths of $10 \mathrm{meV}$.

Since the $L$-valley shunt is expected to result in faster intersubband relaxation, we take $\tau_{12}$ to be roughly a factor of 3 smaller than in the isolated system without the momentumspace reservoir, i.e., $\tau_{\Gamma} / \tau_{12} \approx 0.3$. Following multiplication of $I_{1 \mathrm{~s}}$ in $(2.3)$ by $N / N_{\Gamma} \approx 10$, we find $I_{1 \mathrm{~s}} \approx 54 \mathrm{MW} / \mathrm{cm}^{2}$, implying that saturation has been delayed nearly until the plasma-breakdown point. Noting that $\eta_{s}$ in (2.6) should also be multiplied by $N / N_{\Gamma}$, we obtain $\eta_{s}=7.1$. When this value is employed in the propagation equations (accounting for both $\Gamma$-point and $L$-point absorption), we obtain a maximum conversion efficiency of $\approx 0.20$ at $I_{1}^{\text {in }}=I_{1 s}$. This is a factor of 2 larger than the $\eta_{\max }$ calculated for the same $\Gamma$-valley subband system in the absence of the reservoir (compare also with the results from Section III). Furthermore, since the saturation intensity has just barely been reached, the optimum sample thickness is only slightly greater than $\cos \theta / \alpha_{12}^{0}$. Assuming $\alpha_{12}^{0}=1000 \mathrm{~cm}^{-1}$, we obtain $L_{\max }=$ $7.4 \mu \mathrm{m}$, which is quite feasible for growth by MBE. While the final optimization of $\eta_{\max }$ should again probably involve some detuning from the double resonance condition, here there is less flexibility since one is under the additional constraint of assuring that the $L$-point absorption remain suppressed.

We, thus, predict that the incorporation of a momentumspace reservoir will significantly enhance the performance of an intersubband second-harmonic-generation device. If the goal is to maximize the output power at the secondharmonic wavelength, surface-incidence geometry will provide far greater coupling to a large-area input beam than would be achievable using waveguide geometry. The present analysis demonstrates the feasibility of attaining high conversion efficiencies from surface-incidence devices with practical active-layer thicknesses. While we have illustrated the concept with the example of an InAs-GaSb-AlSb asymmetric double well, similar behavior is expected for $\mathrm{Al}_{x} \mathrm{Ga}_{1-x}$ As-based structures with $L$-point or $X$-point reservoirs.

\section{CONCLUSION}

We have analyzed the prospects for obtaining high secondharmonic conversion efficiencies from semiconductor devices based on interactions between the quantized subbands in asymmetric stepped or double quantum wells. Results for $\eta_{\max }$ and $L_{\max }$ have been derived from solutions to the coupled propagation and transition-rate equations, accounting for absorption, saturation of both the absorption and secondharmonic generation coefficients, and thermal redistribution of the equilibrium subband populations due to optical heating. The numerical calculations confirm the importance of the key figure of merit $\eta_{s}$ (roughly the conversion efficiency at the onset of saturation), which depends only on ratios involving the linewidth, the intersubband relaxation time, and the optical matrix elements. Since there are fundamental limits to the values of those ratios, it becomes clear why conversion efficiencies exceeding $10 \%$ may never be attained in devices based on "conventional" subband systems of the type studied thus far. For surface incidence, even efficiencies approaching that value will require extremely thick active regions, which would be impractical to grow by current methods. While detuning from the double resonance condition is found to be advantageous, single resonance yields relatively modest improvements and non-resonance requires that the active-layer be even thicker.

Our analysis clarifies that the fundamental limits to device performance are effectively independent of such parameters 
as the doping density, angle of incidence, fill factor, refractive index, etc. One is also significantly constrained with regard to optimization of the matrix elements through modification of the asymmetric quantum structure (e.g., in maximizing $R_{12}$ the ratio $z_{13} / z_{12}$ should not be increased beyond unity [10]). It is therefore concluded that efforts to improve $\eta_{\max }$ should probably center on increasing the intersubband relaxation rate or otherwise delaying the onset of saturation. We have proposed to accomplish both of these objectives by placing the subband system in contact with an optically-inactive momentum-space reservoir. A specific device based on $\Gamma$-valley active states and $L$-valley reservoir states in InAs-GaSb-AISb asymmetric double quantum wells is modeled using an 8-band finiteelement algorithm to obtain the band structure and optical matrix elements. The nonlinearity is predicted to saturate only at intensities approaching the damage threshold, and a conversion efficiency of $20 \%$ is obtained for an active layer thickness of less than $10 \mu \mathrm{m}$. The realization of such characteristics would considerably enhance the attractiveness of intersubband second-harmonic devices for the generation of high-intensity coherent pulses in the mid-wave infrared.

\section{ACKNOWLEDGMENT}

The authors thank H. Xie for valuable discussions of intersubband optical properties and Quantum Semiconductor Algorithms for the use of finite-element band structure software.

\section{REFERENCES}

[1] M. K. Gurnick and T. A. DeTemple, "Synthetic Nonlinear Semiconductors," IEEE J. Quantum Electron., vol, QE-19, pp. 791-794, 1983

[2] Z. Ikonic, V. Milanovic, and D. Tjapkin, "Resonant second harmonic generation by a semiconductor quantum well in electric field," IEEE $J$. Quantum Electron., vol. 25, pp. 54-60, 1989

[3] M. M. Fejer, S. J. B. Yoo, R. L. Byer, A. Harwit, and J. S. Harris, Jr., "Observation of extremely large quadratic susceptibility at $9.6-10.8 \mu \mathrm{m}$ in electric-field-biased AlGaAs quantum wells," Phys. Rev. Lett, vol. 62, pp. 1041-1044, 1989

[4] J. Khurgin, "Second-order intersubband nonlinear-optical susceptibilities of asymmetric quantum-well structures," J. Opt. Soc. Am. B, vol. 6, pp. $1673-1682,1989$

[5] P. Boucaud and F. H. Julien, "Generation de second-harmonique dans les puits quantiques asymetriques GaAs-AlGaAs," J. Phys. III, vol. 1 , pp. 13-28, 1991 .

[6] P. Boucaud, F. H. Julien, D. D. Yang, J.-M. Lourtioz, E. Rosencher, P. Bois, and J. Nagle, "Detailed analysis of second-harmonic generation near $10.6 \mu \mathrm{m}$ in GaAs/AlGaAs asymmetric quantum wells," Appl. Phys. Lett., vol. 57, pp. 215-217, 1990.

[7] P. Boucaud, F. H. Julien, D. D. Yang, J.-M. Lourtioz, E. Rosencher, and P. Bois, "Saturation of second-harmonic generation in GaAs-AlGaAs asymmetric quantum wells," Opt. Lett., vol. 16, pp. 199-201, 1991.

[8] E. Rosencher and Ph. Bois, "Model system for optical nonlinearities: Asymmetric quantum wells," Phys. Rev. B, vol. 44, pp. 11315-11327, 1991.

[9] C. Sirtori, F. Capasso, D. L. Sivco, S. N. G. Chu, and A. Y. Cho, "Observation of large second order susceptibility via intersubband transitions at $\lambda \sim 10 \mu \mathrm{m}$ in asymmetric coupled AlInAs/GalnAs quantum wells," Appl. Phys. Lett., vol. 59, pp. 2302-2304, 1991.

[10] E. Rosencher, "Two-photon optical nonlinearities in a resonant quantum well system," J. Appl. Phys., vol. 73, pp. 1909-1911, 1993.

[11] Z. Chen, M. Li, D. Cui, H. Lu, and G. Yang, "Surface-emitting secondharmonic generator by intersubband transition in asymmetric quantum wells with slab waveguide," Appl. Phvs. Lett., vol. 62, pp. 1502-1503, 1993.

[12] T. A. DeTemple, L. A. Bahler, and J. Osmundsen, "Semiclassical theory of resonant three-wave parametric interactions: Second-harmonic generation," Phys. Rev. A, vol. 24, pp. 1950-1964, 1981.
[13] Y. R. Shen, The Principles of Nonlinear Optics. New York: Wiley, 1984.

[14] H. Xie, W. I. Wang, J. R. Meyer, and L. R. Ram-Mohan, "Normal incidence second-harmonic generation in L-valley $\mathrm{AlSb} / \mathrm{GaSb} / \mathrm{Ga}_{1-x} \mathrm{Al}_{x}$ $\mathrm{Sb} / \mathrm{AlSb}$ stepped quantum wells," Appl. Phys. Lett., vol. 65, pp. 2048-2050, 1994.

[15] L. C. West and S. J. Eglash, "First observation of an extremely largedipole infrared transition within the conduction band of a GaAs quantum well," Appl. Phys. Lett., vol. 46, pp. 1156-1158, 1985.

[16] A. Yariv, Quantum Electronics, 2nd ed. New York: Wiley, 1975, ch. 16.

17] P. J. Price, "Two-dimensional electron transport in semiconductor layers. I. Phonon scattering," Ann. Phys., vol. 133, pp. 217-239, 1981

[18] R. J. Bauerle, T. Elsaesser, W. Kaiser, H. Lobentanzer, W. Stolz, and K. Ploog, "Picosecond infrared spectroscopy of hot carriers in a modulation-doped $\mathrm{Ga}_{0.47} \operatorname{In}_{0.53}$ As multiple-quantum-well structure," Phys. Rev. B, vol. 38, pp. 4307-4310, 1988.

[19] H. T. Grahn, H. Schneider, W. W. Ruhle, K. von Klitzing, and K. Ploog, "Nonthermal occupation of higher subbands in semiconductor superlattices via sequential resonant tunneling," Phys. Rev. Lett., vol. 64 , pp. 2426-2429, 1990.

[20] J. Faist, F. Capasso, C. Sirtori, D. L. Sivco, A. L. Hutchinson, S. N. G. Chu, and A. Y. Cho, "Measurement of the intersubband scattering rate in semiconductor quantum wells by excited state differential absorption spectroscopy," Appl. Phys. Lett., vol. 63, pp. 1354-1356, 1993.

[21] F. H. Julien, J.-M. Lourtioz, N. Herschkorn, D. Delacourt, J. P. Pocholle, M. Papuchon, R. Planel, and G. Le Roux, "Optical saturation of intersubband absorption in GaAs- $\mathrm{Al}_{2} \cdot \mathrm{Ga}_{1}{ }_{x}$ As quantum wells," Appl. Phys. Lett., vol. 53, pp. 116-118, 1988.

[22] A. Szilagyi, A. Hordvik, and H. Schlossberg, "A quasi-phase-matching technique for efficient optical mixing and frequency doubling," $J$. Appl. Phys., vol. 47, pp. 2025-2032, 1976.

[23] P. von Allmen, M. Berz, G. Petrocelli, F.-K. Reinhart, and G. Harbeke, "Inter-subband absorption in GaAs/AlGaAs quantum wells between $4.2 \mathrm{~K}$ and room temperature," Semiconduct. Sci. Technol., vol. 3, pp. 1211-1216, 1988.

[24] H. Asai and Y. Kawamura, "Temperature dependence of intersubband absorption in InGaAs/InAlAs multiquantum wells," J. Appl. Phys., vol. 68, pp. $5890-5892,1990$.

[25] M. Zaluzny, "Intersubband absorption line broadening in semiconductor quantum wells: Nonparabolicity contribution," Phys. Rev. B, vol. 43, pp. $4511-4514,199$ J.

[26] F. Muller. V. Petrova-Koch, M. Zachau, F. Koch, D. Grutzmacher, R. Meyer, H. Jurgensen, and P. Balk, "Resonance spectroscopy of InGaAs/InP quantum well sub-bands," Semicond. Sci. Technol., vol. 3, pp. 797-801, 1988

[27] J. Y. Andersson and G. Landgren, "Intersubband transitions in single $\mathrm{AlGaAs} / \mathrm{GaAs}$ quantum wells studied by Fourier transform infrared spectroscopy," J. Appl. Phys., vol. 64, pp. 4123-4127, 1988.

[28] A. Kastalsky, "Infrared intraband laser induced in a multiple-quantumwell interband laser," IEEE J. Quantum Electron., vol. 29, pp. $1112-1115,1993$

[29] B. K. Ridley, "Electron scattering by confined LO polar phonons in a quantum well," Phys. Rev. B, vol. 39, pp. 5282-5286, 1989.

[30] A. Seilmeier, H.-J. Hubner, G. Abstreiter, G. Weimann, and W. Schlapp, "Intersubband relaxation in GaAs- $\mathrm{Al}_{\lambda} \mathrm{Ga}_{1-\gamma}$ As quantum well structures observed directly by an infrared bleaching technique," Phys. Rev. Lett., vol. 59, pp. 1345-1348, 1987.

[31] M. C. Tatham, J. F. Ryan, and C. T. Foxon, "Time-resolved Raman measurements of intersubband relaxation in GaAs quantum wells," Phys. Rev. Lett., vol. 63, pp. 1637-1640, 1989.

[32] U. Plodereder, T. Dahinten, A. Seilmeier, and G. Weimann, "Influence of doping concentration on intersubband relaxation in modulation-doped quantum well structures," Phys. Stat. Sol. (b), vol. 173, pp. 373-379, 1992.

[33] J. K. Jain and S. Das Sarma, "Role of discrete slab phonons in carrier relaxation in semiconductor quantum wells," Phys. Rev. Lett., vol. 62, pp. 2305-2308, 1989

[34] J. L. Educato and J. P. Leburton. "Intersubband relaxation in modulation-doped multiple-quantum-well structures," Phys. Rev. B, vol. 49, pp. 2177-2180, 1994.

[35] G. Sun and J. B. Khurgin, "Optically pumped four-level infrared laser based on intersubband transitions in multiple quantum wells: Feasibility study," IEEE J. Quantum Electron., vol. 29, pp. 1104-1111, 1993.

[36] D. J. Erskine, A. J. Taylor, and C. L. Tang, "Femtosecond studies of intraband relaxation in GaAs, AlGaAs, and GaAs/AlGaAs quantum well structures," Appl. Phys. Lett., vol. 45, pp. 54-56. 1984 
[37] W.-Z. Lin, R. W. Schoenlein, J. G. Fujimoto, and E. P. Ippen, "Femtosecond absorption saturation studies of hot carriers in GaAs and A1GaAs," IEEE J. Quantum Electron., vol. 24, pp. 267-275, 1988.

[38] P. C. Becker, H. L. Fragnito, C. H. Brito Cruz, J. Shah, R. L. Fork, J. E. Cunningham, J. E. Henry, and C. V. Shank, "Femtosecond intervalley scattering in GaAs," Appl. Phys. Lett., vol. 53, pp. 2089-2090, 1988.

[39] W. Hackenberg and G. Fasol, "Determination of the LO-phonon and $\Gamma \rightarrow L$ intervalley scattering time in GaAs from hot electron luminescence spectroscopy," Solid-State Electron., vol. 32, pp. 1247-1251, 1989

[40] S. M. Goodnick and J. E. Lary, "Monte Carlo studies of intersubband relaxation in semiconductor microstructures," Semiconduct. Sci. Technol. vol. 7, pp. B109-115, 1992.

[41] L. C. Lew Yan Voon, L. R. Ram-Mohan, H. Luo, and J. K. Furdyna, "Barrier localization effects in $\mathrm{Al}_{x} \mathrm{Ga}_{1-x} \mathrm{As}_{-} \mathrm{Al}_{y} \mathrm{Ga}_{1-y} \mathrm{As}$ superlattices," Phys. Rev. B, vol. 47, pp. 6585-6589, 1993.

[42] L. C. Lew Yan Voon and L. R. Ram-Mohan, "Band-to-band lasing in type-II GaAs/AlAs short-period superlattices," Superlatt. Microstruct. vol. 14, pp. 49-52, 1993.

[43] C. Parks, A. K. Ramdas, M. R. Melloch, and L. R. Ram-Mohan, "Piezomodulated reflectivity study of minibands in $\mathrm{Al}_{x} \mathrm{Ga}_{1-x} \mathrm{As} / \mathrm{GaAs}$ superlattices," Phys. Rev. B, vol. 48, pp. 5413-5421, 1993.

[44] H. Xie and W. I. Wang, "Normal incidence infrared modulator using direct-indirect transitions in GaSb quantum wells," Appl. Phys. Lett., vol. 63, pp. 776-778, 1993.

[45] O. Madelung, Ed., Landbolt-Börnstein, Numerical Data and Functional Relationships in Science and Technology. Berlin: Springer, 1982, Gp. III, vol. 17.

[46] J. Menendez, A. Pinczuk, D. J. Werder, J. P. Valladares, T. H. Chiu, and W. T. Tsang, "Band lineups at the GaSb-Al $\mathrm{Ga}_{1-x} \mathrm{Sb}$ heterojunction: Experimental evidence for a new common anion rule," Solid State Commun., vol. 61, pp. 703-706, 1987.

[47] G. J. Gualtieri, G. P. Schwartz, R. G. Nuzzo, R. J. Malik, and J. F. Walker, "Determination of the (100) InAs/GaSb heterojunction valence- band discontinuity by $\mathrm{x}$-ray photoemission core level spectroscopy," $J$ Appl. Phys., vol. 61, pp. 5337-5341, 1987.

[48] C. Alibert, A. Joullie, A. M. Joullie, and C. Ance, "Modulationspectroscopy study of the $\mathrm{Ga}_{1-}{ }_{x} \mathrm{Al}_{x} \mathrm{Sb}$ band structure," Phys. Rev. B vol. 27 , pp. $4946-4954,1983$.

J. R. Meyer, photograph and biography not available at the time of publication.

C. A. Hoffman, photograph and biography not available at the time of publication.

F. J. Bartoli, photograph and biography not available at the time of publi cation.

E. R. Youngdale, photograph and biography not available at the time of publication.

L. R. Ram-Mohan, photograph and biography not available at the time of publication 